\title{
A preliminary communication on whether general dental practitioners have a role in identifying dental patients with mental health problems
}

\author{
F. Lloyd-Williams, ${ }^{1}$ C. Dowrick, ${ }^{2}$ D. Hillon, ${ }^{3}$ G. Humphris, ${ }^{4}$ G. Moulding, ${ }^{5}$ and R. Ireland ${ }^{6}$
}

\begin{abstract}
Objective To examine whether general dental practitioners regard themselves as having a role in identifying dental patients with mental health problems.

Method Telephone interviews were conducted with, and postal questionnaires were sent to, a random sample $(n=94)$ of general dental practitioners on Health Authority lists within the Mersey Region. A total of 84 general dental practitioners responded, resulting in an $89 \%$ response rate.

Results The majority of general dental practitioners had encountered patients with mental health problems $(78 \%$ of interviewees, $56 \%$ of questionnaire responders). However, nearly half of the interviewees $(46 \%)$ do not refer patients with mental health problems. When referrals to dental specialists were made, they tended to be regarding a physical manifestation rather than a psychological one. The majority of interviewees and questionnaire responders (55\% and $82 \%$ respectively) expressed a 'positive' response to the development of a referral role. Nevertheless, there were some reservations concerning the practicalities of its development and implementation. Conclusion The majority of general dental practitioners consider a role in identifying patients with possible mental health problems. This role might include, being able to identify patients with undiagnosed mental health problems, and being aware of dental conditions which may be caused by mental health problems. To achieve this, inter-professional co-operation between general dental practitioners and other health professionals requires development.
\end{abstract}

Studies have shown that symptoms of anxiety and depression are common in the community. In a study of 10,000 adults in the UK, Meltzer et al. ${ }^{1}$ found a $16 \%$ point prevalence of psychiatric morbidity, of which mixed anxiety and depressive disorders (8\%) were the most common. In primary medical care settings the prevalence of total psychiatric morbidity is substantially higher, in the order of

\footnotetext{
$1^{*}$ Research Associate, Department of Primary Care, Liverpool University. ${ }^{2}$ Professor of Primary Medical Care, Department of Primary Care, Liverpool University. ${ }^{3}$ Dental Practitioner, Jones and Hillon Dental Practice, Netherton, Liverpool. ${ }^{4}$ Senior Lecturer, Department of Clinical Psychology, Liverpool University. ${ }^{5}$ Dental Practitioner, Pooltown Dental Practice, Ellesmere Port, Wirral. ${ }^{6}$ Course Director, Department of Clinical Dental Sciences Liverpool University.

${ }^{*}$ Correspondence to: Ffion Lloyd-Williams, Department of Primary Care, Liverpool University, Whelan Building (2nd Floor), Quadrangle, Brownlow Hill, Liverpool L69 $3 G B$

REFEREED PAPER

Received 05.03.01; Accepted 16.07.01

(C) British Dental Journal 2001; 191: 625-629
}

23-25\%. ${ }^{2}$ Friedlander et $\mathrm{l}^{3}$ stated that dentists should be concerned with the identification of patients with depression due to its extensive association with dental disease. Studies have found that dental fear may represent an underlying phobia or trait anxiety. ${ }^{4,5}$ Stress associated with financial strain that manifests as depression are significant risk indicators for more severe periodontal disease. ${ }^{6}$ Of patients with facial pain syndromes, $57 \%$ have a mental illness. ${ }^{7,8}$ 'Burning mouth syndrome' (BMS) is also thought to have mental health disorders as one of its origins, ${ }^{9-12}$ and the sensation of oral dryness might be a psychophysiological expression of depression. ${ }^{13,14}$ It has been suggested that psychiatric distress may be as common in general dental practice as in general medical practice. ${ }^{15}$ To date, little is known about the presence of anxiety and depression amongst primary dental care patients. Although reports have shown that dentists are able to recognise mental health problems in their patients, ${ }^{16,17}$ there is an apparent lack of training in helping dentists to assist their patients with mental health problems.

The aim of the study was to determine:

1. To what extent do general dental practitioners (GDPs) encounter dental patients with mental health problems?

2. What procedure do GDPs currently adopt with such dental patients?

3. Do GDPs regard themselves as having a role in identifying dental patients with mental health problems?

\section{Method}

Figure 1 summarises the profile of the sample. Telephone interviews were conducted with, and postal questionnaires sent to, a random sample $(n=94)$ of all GDPs on the health authority lists within the Mersey region. In total 54 GDPs agreed to a telephone interview thus resulting in a response rate of $57 \%$. A key reason given for not being able to participate in a telephone interview was lack of time. In an attempt to gain the opinion of these GDPs $(n=40)$, a semistructured postal questionnaire, based upon the questions included in the telephone interview, was sent with a reply paid envelope. This approach resulted in the return of 30 questionnaires, representing a $75 \%$ response rate. The overall response from the eligible sample of 94 GDPs was $89 \%$.

The data derived from the telephone interviews was superior to the questionnaire responses due to the ability to explore the GDPs replies. Nonetheless, the questionnaire allowed the provision of additional opinions which otherwise would have been missed, and enabled verification of the telephone interview data.

All telephone interviews with GDPs were audiotaped and each individual interview was transcribed. Each individual transcript was scrutinised to identify key issues, concepts and themes, which were coded and then categorised accordingly. These categories were 


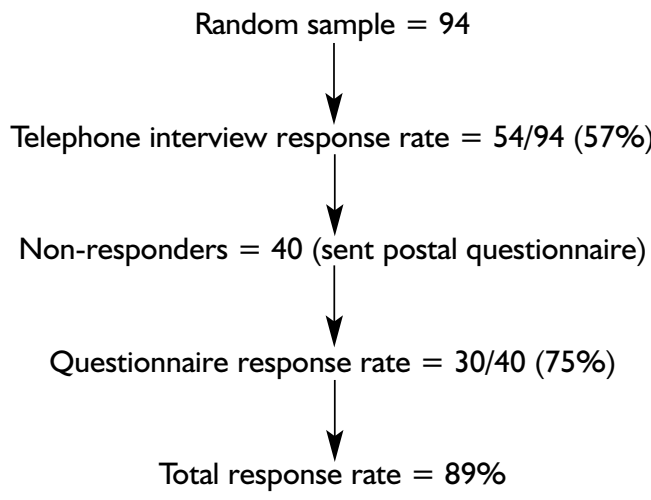

Figure I. Profile of random sample of general dental practitioners

expanded with new transcripts and refined as new themes emerged. The data was examined for both similarities and differences within themes. The context of the interview and characteristics of the interviewees were retained, thus helping to understand, interpret and explain the findings.

All demographic data and the quantitative data obtained via the questionnaires returned by GDPs were analysed by SPSS for Windows V10. Frequencies and cross-tabulations were performed.

The reliability of the qualitative data analysis was enhanced by the independent investigation of a cross-section of the transcripts, by the 'dental project team'. The team comprised 2 GDPs, 1 sociologist, 1 dental care specialist, 1 medical care specialist and 1 clinical psychologist. From this, level of agreement was assessed. The validity of the findings was ensured by adopting the 'triangulation' approach to data collection. ${ }^{18}$ Furthermore, 'negative' and 'deviant' cases are reported in the findings, thereby ensuring a thorough interpretation of the obtained data.

Appropriate ethical committee approval was obtained.

\section{Results}

Table 1 summarises the gender profile and years in practice for the GDPs who participated in the telephone interview or responded to the questionnaire. The gender ratio of the study represents that of the general GDP population. A cross-section of GDPs, in terms of time in practice, was also attained. The majority of respondents had been practising GDPs for at least 11 years.

Table I. Gender profile and years in practice for random sample of GDPs Telephone Interview Postal Questionnaire Profile of Respondents(\%) Respondents (\%) Non-Responders(\%)

\begin{tabular}{lccc}
\hline $\begin{array}{l}\text { Gender } \\
\text { Male }\end{array}$ & $42(78)$ & $24(80)$ & $10(91)$ \\
Female & $12(22)$ & $6(20)$ & $\mathrm{I}(9)$ \\
& & & \\
\hline $\begin{array}{l}\text { Years of Practice } \\
\text { Over 20 years }\end{array}$ & $25(46)$ & $14(47)$ & $4(36)$ \\
$\begin{array}{l}\text { II-20 years } \\
\text { less than 10 years }\end{array}$ & $10(35)$ & $16(53)$ & $5(45)$ \\
\hline Total & 54 & - & $2(19)$ \\
\hline
\end{tabular}

It was found that GDPs encounter dental patients with mental health problems. These encounters range from dental anxiety/fear to severe mental illness. In between these two points, there are patients with anxiety and depression due to life events, dental somatisation disorders (i.e. the patient presents with dental pain, but the GDP can find no physical cause), patients with behaviour suggesting a psychological disorder, and patients with diagnosed psychological problems. The relevant findings are summarised in Table 2.
Table 2. Categorisation of GDP encounters with patients with possible mental health problems

\begin{tabular}{|c|c|c|}
\hline \multirow[b]{2}{*}{$\begin{array}{l}\text { Nature of mental } \\
\text { health problem }\end{array}$} & \multicolumn{2}{|c|}{ Encounters* } \\
\hline & $\begin{array}{c}\text { Telephone Interview } \\
\text { Respondents } \\
n=42(78 \%)\end{array}$ & $\begin{array}{c}\text { Questionnaire } \\
\text { Respondents } \\
\mathrm{n}=17(57 \%)\end{array}$ \\
\hline Dental anxiety/fear & $21 \%$ & $18 \%$ \\
\hline $\begin{array}{l}\text { Anxiety/depression due } \\
\text { to life events }\end{array}$ & $36 \%$ & $12 \%$ \\
\hline $\begin{array}{l}\text { Dental somatisation } \\
\text { disorders }\end{array}$ & $62 \%$ & $53 \%$ \\
\hline $\begin{array}{l}\text { Behaviour suggesting a } \\
\text { psychological disorder }\end{array}$ & $33 \%$ & - \\
\hline $\begin{array}{l}\text { Diagnosed psychological } \\
\text { problems }\end{array}$ & $48 \%$ & - \\
\hline Severe mental illness & $12 \%$ & $6 \%$ \\
\hline
\end{tabular}

* Some GDPs mentioned more than one encounter.

Questionnaire respondents reported fewer encounters than telephone interviewees. This is possibly due to the different data collection technique employed. Whereas the elaboration and explanation of the question posed was possible with the telephone interviews, no such opportunity existed with the questionnaire. This may have resulted in the under-reporting of encounters by questionnaire respondents.

Just over half of the questionnaire respondents (57\%) claimed to have encountered patients with symptoms they suspected were caused by mental health problems. Their responses indicated that the majority of encounters were with patients who had a 'dental somatisation disorder' (53\%) evidenced by such comments as:

The patient was convinced that all his teeth needed extracting in order to cure his headaches.

The majority of GDPs interviewed (78\%) stated they had encountered patients who had been diagnosed, or were possibly suffering from mental health problems (undiagnosed). Of the 12 GDPs who stated they were unaware of such patients, eight conceded this was due to a lack of knowledge/skills to identify such patients. The following comment was typical:

I'm sure I do encounter patients with psychological problems - but I wouldn't say I was an expert at spotting them.

Table 3 summarises the current procedure adopted by GDPs. Nearly half of all GDPs interviewed (46\%) do not refer patients they suspect of having mental health problems. Even when GDPs do refer their patients to dental specialists, it tends to be regarding a physical manifestation as opposed to a psychological one.

In circumstances where the GDPs do not refer dental patients with mental health problems, some suggested they allocate extra time to treat the patient.

Two GDPs admitted to administering unnecessary dental treatment in order to satisfy patients' unusual requests. In a similar manner, another GDP stated that he would 'pretend' to administer treatment as well as administering unnecessary treatment:

I actually have a patient at the moment where there doesn't seem to be any cause for his problem, but he always comes in saying 'I've got pain in this area. What I do is lengthen my appointments with him and I give him the impression that I am treating him... In extreme cases, I might take out a filling and replace it if a patient really is adamant...

Table 3. Categorisation of GDPs' current procedure with patients with possible mental health problems

Current procedure Number of interviewees (\%)

GDP adapts treatment accordingly $25(46)$

Referral to dental specialists $\quad 17(32)$

Referral to GMP $5(9)$

No such patients encountered $\quad 7(13)$ 


\section{RESEARCH mental health}

This suggests that the true aetiology of the patients' problems may not always be addressed. At best, the procedure being adopted by the GDPs is an inefficient use of their time, and at worst, the patients are receiving inappropriate intervention.

All the GDPs who perceived the patient to have poor mental health focused on managing the physical symptoms of the dental condition. Although some GDPs (9\%) indicated that they 'talk' to their patients about their problem, they stop short of addressing the underlying problems.

GDPs adopted one of two approaches when referring a patient to a dental specialist. A small number of GDPs (29\%) stated they would provide some basic treatment and then refer the patient. The remaining GDPs (71\%) indicated they would refer such patients to a dental specialist without attempting to administer treatment.

Only five GDPs (9\%) stated that they referred dental patients with possible mental health problems to their general medical practitioner (GMP). In general, GDPs appeared cautious of GMPs' reaction to the idea of identifying dental patients with possible mental health problems.

Seven GDPs (13\%) stated that they did not encounter patients with mental health problems. However, the comments made by these GDPs indicate they do not look for, or necessarily recognise the symptoms and signs of psychological difficulties.

The responses received from the questionnaire responders served as verification of the findings from the interviews with GDPs. Of the $57 \%$ of questionnaire responders who encountered patients with possible mental health problems, $29 \%$ of GDPs stated that they did not refer patients, with $12 \%$ of GDPs stating they spent time 'listening' to the patients. The majority (65\%) refer their patients to another health professional. However the referrals are, in the majority of cases, in relation to the physical dental problems, as opposed to the potential underlying mental health problems. Only $12 \%$ of GDPs stated a referral would be to the patient's GMP.

As shown in Table 4, the majority of interviewees (55\%) expressed a 'positive' response to the development of a referral role for patients with possible mental health problems. It should not be ignored however that a sizeable minority $(n=16,30 \%)$ were uncertain about developing such a role. Although there was a positive outlook towards such a procedure, some GDPs still had reservations concerning the practicalities of its development and implementation.

$\begin{aligned} & \text { Table 4. GDPs' response to the future referral of patients with mental } \\
& \text { health problems }\end{aligned}$
\begin{tabular}{lc}
\hline GDPs' response & Number of interviewees (\%) \\
\hline Positive & $30(55)$ \\
Uncertain & $16(30)$ \\
Negative & $8(15)$ \\
Total & $\mathbf{5 4}(\mathbf{1 0 0})$
\end{tabular}

The majority of questionnaire responders ( $n=14 / 17,82 \%)$ indicated they would refer patients if suitable services were available. Of the five GDPs who did not currently refer patients, four stated they would refer such patients if 'appropriate locations were available' (i.e. clinics, possibly based at a general practice; cognitive behaviour therapy; psychosocial counselling). They felt that such facilities/appropriate referral locations were not currently available to them. Of the remaining nine GDPs, five indicated the need for an identified clinic/unit with a counsellor/psychiatrist. Nevertheless, some concern was raised regarding dental patients' response to such a referral. It was thought that the stigma of mental health problems may cause reluctance to accept such a referral. A suggestion for overcoming this problem was to improve GDPs skills to broach the issue.

The majority of interviewed GDPs (70\%) felt the patient's GMP was a good place for initial referral. Their comments echo the observations made earlier that GMPs are in a position to make a qualified assessment of a patient's mental health, based upon the GDP's opinion.

However, collaboration between GDPs and GMPs was not currently prevalent, with $30 \%$ of GDPs commenting that there was definite need for improvement in the way GDPs and GMPs communicate. As one GDP put it:

'We're all in our own little boxes and we don't really communicate with one another, it's probably historical barriers. I think it is an educational thing, if we were taught to communicate more, at university maybe, things would be better.'

Barriers to communication included the vicinity of dental practices to general medical practices, a lack of appreciation of professional roles, and not being able to speak directly to GMPs.

Overall, GDPs who wished to develop their role in the identification and referral of patients with mental health problems, seemed most willing to do so with GMPs.

The reasons for GDPs expressing uncertainty or negativity towards identifying and referring dental patients with mental health problems was due to their scepticism of GMPs accepting them in this role and the reaction of dental patients.

Concerns about the patient's reaction to raising the issue of being referred for mental health problems were expressed by half of the GDPs ( $\mathrm{n}=8 / 16,50 \%)$. The following response was typical:

'It sounds wonderful, yes, but I must admit I would be a bit unsure how to broach it with a patient. There is still this stigma isn't there.'

However, as implied by one of the GDPs who welcomed the idea of developing a referral role, the important factor is the manner adopted with the dental patient. It requires a degree of skill upon the part of the GDP and trust on the part of the patient. As the GDP put it:

'You have to put it in a careful and caring way and ensure the relationship is good. You've got to do it very carefully and build up a relationship first and then, may be after a few appointments, you could say, 'hey you know, I know someone else who was like you and they became much happier once they saw so and so'. I don't think you could do it with a new patient...'

The remaining $50 \%$ of GDPs expressed concern about how GMPs would react to them adopting such a role. As previously observed, there is currently, from the viewpoint of GDPs at least, a distinct lack of communication between themselves and GMPs. GDPs feel that they are regarded by GMPs as being only concerned with oral health.

Of the eight GDPs who gave a negative response to developing a referral role, seven of them stated that this was due to their reluctance to refer to GMPs. This reluctance was due to previous experiences where GDPs had attempted to enlist the help of a GMP regarding dental practice patients who appeared to be experiencing mental health problems.

\section{Discussion}

It would appear that the majority of GDPs consider having a role in identifying patients with possible mental health problems. More specifically, the GDPs role might include:

(1) being able to identify people whose mental health problems have so far been undiagnosed.

(2) being aware of the dental conditions which may be caused by or associated with mental health problems.

Many GDPs are able to recall patients that have presented with symptoms which are either perceived as unsuitable for treatment or are suspected as being psychological in origin, but in the majority of cases GDPs do not refer. Although some GDPs attempt to adapt their approach and treatment according to the nature of the mental health problem with which they are presented, this appears to inadequately address the needs of these dental patients. They tend to treat the physical condition and ignore the underlying problem 
even though they admit that this may not be the best course of action. In many instances this was due to not knowing where to refer the patient or how to broach the subject of referral with the patient.

The reason for referral given by GDPs to the dental specialist often related to a patient's oral condition as opposed to possible underlying mental health problems. It is therefore questionable whether the referral being made was necessary or appropriate. Firstly, if no physical problem was identified, what happened to the patient thereafter? No GDPs reported any feedback, although many of the GDPs speculated that such patients just became 'lost in the system'. If the psychological problem was not detected, it can only be assumed that it was not treated. Secondly, is this approach an effective use of health service resources? If the true problem were psychological rather than physical, a referral direct to a patient's general medical practitioner (GMP) or to the appropriate mental health services might be considered more appropriate.

Surprisingly very little communication was reported between GDPs and GMPs considering that both professions are part of the 'primary care team'. It was apparent from the interviews that GDPs currently refer dental patients to GMPs on an 'ad hoc' basis. Three issues emerge from this current practice. 1) There is no guarantee that the dental patient follows the GDP's recommendation and goes to visit their GMP. 2) GDPs do not appear to inform GMPs of the reason for sending the dental patient to see them, therefore the GMP and 'dental patient' consultation may not relate to the problem suspected by the GDP. 3) The GDP does not receive any feedback from the GMP or make any follow up enquiries regarding the outcome of the consultation. Improving methods of information exchange between GDPs and GMPs requires further investigation, however the benefits of increased integration have been previously highlighted. ${ }^{19,20,21}$

In terms of the dental patient's welfare, a referral to the GMP may be the most appropriate option. Based upon the premise that patients may initially be resistant to a referral, referral to the GMP would be the easier option of dealing with a patient presenting with mental health problems, as opposed to suggesting a referral to a psychiatrist. GDPs and GMPs are both part of 'primary care provision' therefore communication and collaboration should be easily developed and dental patients are more likely to accept a referral to their own GMP (i.e. they are likely to be acquainted with them) rather than an unknown health professional. A referral to a GMP would act as confirmation or refutation of the GDPs assessment. This would help to ensure that dental patients with mental health problems were not referred inappropriately (i.e. to mental health services).

Although some GDPs refer patients to a dental specialist/GMP, currently no guidelines or agreed protocol exists which provides GDPs with the assurance that they are identifying and referring patients appropriately. GDPs do not appear to detail in their referral communication their suspicions concerning the possibility of a psychological as opposed to physical origin to the dental complaint. A follow up of referred patients would benefit from further study.

Many GDPs appeared cautious about their ability to determine a patient's psychological condition, this was especially so in the case of depression where it may just be short term (for example, a bad week at work). This caution may be due to a lack of training to identify such problems. Because of potential patient sensitivity, it can be argued that one of the most appropriate locations for a referral would be to the patient's own general practitioner who could then assess the patient's condition and decide upon the best course of treatment. A referral directly to a psychologist may offend or upset the patient, and could also lead to negative consequences for the GDP/patient relationship if no psychological problem was found.
It has to be appreciated that a GDP's workload places a limit upon the amount of time spent with each patient. However, the current procedures adopted for dealing with such patients are ineffective (that is referring for: 'physical' problems, administering unnecessary treatment, having them re-attend). By having set guidelines which outline what symptoms GDPs should be aware of, and when a patient should be referred to their GMP, will not only address the patient's health needs, but also result in an efficient use of time and resources. Obviously, GDPs require a tool that will allow a relatively quick assessment. The hospital anxiety and depression scale ${ }^{22}$ and modified dental anxiety scale ${ }^{23}$ may be useful tools for this procedure.

\section{Conclusion}

This study suggests that:

(1) GDPs are aware of encountering patients with conditions that may not require dental treatment, due to their psychosomatic origin.

(2) The majority of GDPs are willing to help these patients, although currently their referral may be inappropriate due to not having the skills/guidelines to identify the best course of action.

The authors wish to thank Mrs. Margaret Ling who was instrumental in developing the qualitative component of this research study. Also the authors wish to acknowledge the NHS Executive North West Research and Development Directorate for providing the funding to conduct this piece of research.

1. Meltzer H, Gill B, Petticrew M, Hinds K. The prevalence of psychiatric morbidity amongst adults aged 16-64 living in private households in Great Britain. OPCS Surveys of Psychiatric Morbidity in Great Britain Bulletin 1. London OPCS Social Survey Division. 1994.

2. Goldberg D, Huxley P. Common Mental Disorders. London: Routledge. 1992.

3. Friedlander A H, West L J. Dental management of the patient with major depression. Oral Surg Oral Med Oral Path 1991; 71: 573-578.

4. Moore R, Brodsgaard I, Birn H. Manifestations, acquisitions and diagnositc categories of dental fear in a self-referred population. Behaviour Res Ther 1991; 29: 51-60.

5. Milgrom P, Weinstein P, Kleinknecht R, Getz T. Treating fearful dental patients: A clinical handbook. 1985 VA: Reston.

6. Genco R J, Ho A W, Grossi S G, et al. Relationship of stress, distress and inadeqaute coping behaviours to peridontal disease. J Periodont 1999; 70: 711-723.

7. Merskey H, Lau C, Russell E, et al. Screening for psychiatric morbidity. The pattern of psychological illness and morbid characteristics in four chronic pain populations. Pain 1987; 30: 141-157.

8. Feinmann C, Harris M. Psychogenic facial pain. Part 1. Br Dent J 1984; 156: 158-165.

9. Browning S, Hislop S, Scully C, Shirlaw P. The association between burning mouth syndrome and psychosocial disorders. Oral Surg Oral Med Oral Path 1987; 64: 171-144.

10. Grushka M, Sessle B J, Miller R. Pain and personality profiles in burning mouth. Pain 1987; 28: 155-167.

11. van der Ploeg H M, van der Wal N, Eijkman M A J, van der Waal I. Psychological aspects of patients with burning mouth syndrome. Oral Surg, Oral Med Oral Path 1987; 63: 664-668.

12. Zilli C, Brooke R I, Lau C L, Merskey H. Screening for psychiatric illness in patients with oral dysesthesia by means of the general health questionnaire twenty-eight item version (GHQ-28) and the irritability depression and anxiety Scale (IDA). Oral Surg, Oral Med and Oral Path 1989; 67: 384-389.

13. Bergdahl M, Bergdahl J, Johansson I. Depressive symptoms in individuals with idiopathic subjective dry mouth. J Oral Path Med 1997; 26: 448-50.

14. Anttila S S, Knuuttila M L, Sakki T K. Depressive symptoms as an underlying factor of the sensation of dry mouth. Psychosomatic Med. 1998; $60: 215-218$.

15. Green R, Humphris G, Linsay S, Mellor A, Millar K, Sidebotham B. Psychiatric distress, pain and fear in patients in general dental practice. Community Dentistry Oral Epidemiol 1997; 25: 187-188.

16. Baldwin P J, Dodd M, Rennie J S. Improving working conditions and professional support will benefit young dentists. Br Dent J 1999; 186: 30-36.

17. Kells B E, Lime D L, Kenedy J G, Freeman R. Dysmorphophobia: A case successfully treated by a multidisciplinary approach. Dental Update 1996; 23: 402-404. 
18. Denzin N K. The research act. New York: McGraw-Hill, 1978.

19. Mason D K, Gibson J, Devennie J C, Haughney M G, Macpheron L. Integration of primary care dental and medical services: a pilot investigation. Br Dent J 1994; 177: 283-286.

20. Haughney M G, Devennie J C, Macphearson L M, Mason D K. Integration of primary care dental and medical services: a three-year study. Br Dent J 1998; 184: 343-347.

21. Jones R, McConville J, Mason D, Macphearson L, Naven L, McEwen J. Attitudes towards, and utility of, an integrated medical-dental patient-held record in primary care. Br J Gen Pract 1999; 49: 368-373.
22. Zigmond A S, Snaith R P. The hospital anxiety and depression scale. Acta Psychiatricia Scandinavica 1983; 67: 361-370.

23. Humphris G M, Morrison T, Lindsay S J E. The modified dental anxiety scale: validation and United Kingdom norms. Community Dent Health 1995: 12: 143-150.

24. Kendrick T. Depression management clinics in general practice? Br Med J 2000; 320: 527-528.

25. Railton S, Mowat H, Bain J. Optimizing the care of patients with depression in primary care: the views of general practitioners. Health and Social Care in the Community 2000; 8: 119-128. 